\title{
The polydisperse cell model: Non-linear screening and charge renormalization in colloidal mixtures
}

\author{
Aldemar Torres ${ }^{1}$, Gabriel Téllez ${ }^{2}$, René van Roij ${ }^{1}$ \\ 1 Institute for Theoretical Physics, Utrecht University \\ Leuvenlaan 4, 3584 CE Utrecht, The Netherlands. and \\ 2 Departamento de Física, Universidad de Los Andes \\ A. A. 4976 Bogotá, Colombia.
}

(Dated: December 1, 2018)

\begin{abstract}
We propose a model for the calculation of renormalized charges and osmotic properties of mixtures of highly charged colloidal particles. The model is a generalization of the cell model and the notion of charge renormalization as introduced by Alexander and his collaborators (J. Chem. Phys. 80, $5776(1984))$. The total solution is partitioned into as many different cells as components in the mixture. The radii of these cells are determined self-consistently for a given set of parameters from the solution of the non-linear Poisson-Boltzmann equation with appropriate boundary conditions. This generalizes Alexanders's model where the (unique) Wigner-Seitz cell radius is fixed solely by the colloids packing fraction. We illustrate the technique by considering a binary mixture of colloids with the same sign of charge. The present model can be used to calculate thermodynamic properties of highly charged colloidal mixtures at the level of linear theories, while taking the effect of non-linear screening into account.
\end{abstract}

PACS numbers: 82.70.Dd, 64.60.Cn, 64.10.+h, 82.39.Wj

\section{INTRODUCTION}

Typical charge-stabilized colloidal suspensions consist of mesoscopic particles and ions, immersed in a solvent which to a good approximation can be considered as a continuum medium, characterized by a dielectric permittivity $\epsilon$. The microscopic ions form a diffuse layer around the colloidal surfaces, with a typical thickness of the order of the Debye length of the electrolyte $\kappa^{-1}$, and with a net charge that compensates the charge of the colloidal surface. As a consequence, the effective colloidal interactions are screened: instead of the bare Coulomb interaction $Z^{2} e^{2} / \epsilon r$ between two colloidal spheres of radius $a$ and charges $Z e$ a distance $r$ apart in the solvent, standard linear PoissonBoltzmann theory predicts a screened coulomb interaction $Z^{2} e^{2} \exp (2 \kappa a)(1+\kappa a)^{-2} \exp [-\kappa r] / \epsilon r$ between the colloids $[1,2]$. However, it is well-known that this relatively simple picture needs modification in the case of highly charged colloids, where the strong electrostatic coupling between the colloids and their counterions induces the accumulation of the latter close to the colloidsurface. This condensation phenomenon cannot be described by a linearized Poisson-Boltzmann approach, and requires the full nonlinear Poisson-Boltzmann theory for a proper description [3]. However, the simplicity of the linear theories can be retained by the use of the concept of charge renormalization, which considers each colloid and its condensed counterion shell as a single entity, carrying a net effective charge which is usually smaller than the bare (structural) charge $Z$ of the colloid. This socalled renormalized charge, $Z^{\text {ren }}$, characterizes the longdistance behavior of the screened electric potential created by the colloid and its condensed microion polarization cloud, and the effective colloidal pair interaction takes the linear-screening form but with $Z$ replaced by $Z^{\text {ren }}$.

The concept of charge renormalization, and also its relatives such as dressed-ion theory [4], is very welldeveloped by now for monodisperse colloidal suspensions $[3,5-7]$. The traditional theory by Alexander et al. is based on a cell model, in which a suspension of $N$ colloidal spheres in an electrolyte of volume $V$ is approximated by $N$ identical spherical cells of volume $V / N$, each containing a single colloid in the center and co- and counterions such that each cell is neutral. The simple spherically symmetric geometry allows for a straightforward numerical solution of the nonlinear Poisson-Boltzmann equation in the cell, from which $Z^{\text {ren }}$ follows directly $[3,5-7]$. Although such an approach based on cells is mostly justified for colloidal crystals, the concept of charge renormalization has proved to be very useful for fluid-state suspensions, e.g. in describing the structure factor [8] or the electrophoretic mobility [9].

Much less is known about charge renormalization in the case of colloidal mixtures or in polydisperse colloidal suspensions. In recent years such mixtures of charged colloids have generated a lot of interest, for instance because of their ability to form a plethora of crystal phases $[10,11]$ or fluid-fluid demixing phenomena [12]. Moreover, in the presence of external fields colloidal mixtures also show a richer behavior than that of monodisperse systems, e.g. oppositely charged colloids can exhibit lane formation in an electric field [13-15], while sedimentation in the Earth's gravity field can generate layers of colloids with equal mass-per-charge [16] or a "colloidal Brazil nut effect" in which a layer of heavier colloids floats on top of a layer of lighter ones [17]. Given the success of the cell model and of the concept of charge renormalization for the calculation of thermodynamic properties of monodis- 
perse highly charged colloidal suspensions, as well as the recent interest and advances in the synthesization of colloidal mixtures, it is desirable to extend the cell model to the polydisperse domain. The aim of this paper is to introduce such a generalized cell model and to study some of its features, in particular the dependence on the composition of the mixture - this plays of course no role in monodisperse suspensions.

The outline of this paper is as follows. In section 2 we introduce the model. In section 3 we discuss the particular case of a binary mixture. We calculate saturation curves for the renormalized charges, osmotic properties, and the radii of the cells as a function of composition, screening length and total colloid density. Discussion and some general remarks are presented in section 4 , followed by conclusions in section 5 .

\section{MODEL}

Let us begin by considering two species of spherical colloids with charges $Q_{a}=Z_{a} e, Q_{b}=Z_{b} e$ and radius $a$ and $b$, respectively, where $e$ is the proton charge and where we assume that the colloids possess charges of the same sign, i.e., $Z_{a} Z_{b}>0$. Let $N_{a}$ and $N_{b}$ denote the total number of colloids of type $a$ and those of type $b$ respectively. The colloidal mixture occupies a volume $V$ and the colloids are immersed in a medium that is in osmotic equilibrium with a salt reservoir with screening length $\kappa^{-1}=\left(8 \pi \rho_{s} \lambda_{B}\right)^{-1 / 2}$ where $2 \rho_{s}$ is the salt concentration in the reservoir. As usual we have defined the Bjerrum length $\lambda_{B}=\beta e^{2} / \varepsilon$, with $\varepsilon$ the dielectric constant of the solvent and $\beta=1 /\left(k_{B} T\right)$, with $T$ the temperature and $k_{B}$ the Boltzmann constant. The average number densities of colloids are defined by $\rho_{a}=N_{a} / V$ and $\rho_{b}=N_{b} / V$ and their respective volume fractions are $\eta_{a}=4 \pi a^{3} \rho_{a} / 3$ and $\eta_{b}=4 \pi b^{3} \rho_{b} / 3$. The composition of the mixture is characterized by the molar fraction $x=N_{a} /\left(N_{a}+N_{b}\right)$ which in the case of equisized colloids can be written as $x=\eta_{a} / \eta$ with $\eta=\eta_{a}+\eta_{b}$ the total packing fraction of the suspension.

Following the usual cell model approximation we consider the Wigner-Seitz cell of each colloidal particle as a sphere concentric with the spherical colloid. However, due to the difference in size and charge of each species, there is no a priori reason to assume that the cells corresponding to each species are of equal size. Therefore, we suppose that the Wigner-Seitz cells of the colloids of type $a$ have a radius $R_{a}$ and those of the colloids of type $b$ have radius $R_{b}$, which are in principle different and to this point unknown.

Let $\phi_{a}(r)=\beta e \psi_{a}(r)$ be the reduced electric potential inside a cell of type $a$, with $\psi_{a}(r)$ the electric potential at a radial distance $r$ from the center of the colloid. Accordingly, we define $\phi_{b}(r)=\beta e \psi_{b}(r)$ the reduced electric potential inside a cell of type $b$. In the mean-field approximation, the electric potential inside each cell is the solution of the Poisson-Boltzmann equation

$$
\begin{aligned}
\Delta \phi_{a}(r) & =\kappa^{2} \sinh \phi_{a}(r) \\
\Delta \phi_{b}(r) & =\kappa^{2} \sinh \phi_{b}(r)
\end{aligned}
$$

together with the boundary conditions

$$
\begin{aligned}
\left.r \frac{d \phi_{a}}{d r}\right|_{r=a}=-Z_{a} \lambda_{B} / a ;\left.\quad r \frac{d \phi_{b}}{d r}\right|_{r=b} & =-Z_{b} \lambda_{B} / b \\
\left.\frac{d \phi_{a}}{d r}\right|_{r=R_{a}}=0 ;\left.\quad \frac{d \phi_{b}}{d r}\right|_{r=R_{b}} & =0
\end{aligned}
$$

which are obtained from direct application of Gauss' law at the colloid surface (Eq. (2)), and at the boundary of the cell (Eq. (3)). The latter equation accounts for the electroneutrality of each cell. These equations completely determine the electrostatic potential inside each cell. However, the radius of each cell $R_{a}$ and $R_{b}$ remain undetermined, so far. These are determined by the following relations:

$$
\eta_{a} \frac{R_{a}^{3}}{a^{3}}+\eta_{b} \frac{R_{b}^{3}}{b^{3}}=1,
$$

which expresses the fact that the Wigner-Seitz cells fill the whole accessible volume $V$, and

$$
\phi_{a}\left(R_{a}\right)=\phi_{b}\left(R_{b}\right)
$$

which is a consequence of the continuity of the electric potential, and the fact that cells of different species can be in contact with each other. The system of differential and algebraic equations (1), (2), (3), (4) and (5) is a complete set to determine the unknown parameters and functions $R_{a}, R_{b}, \phi_{a}(r)$ and $\phi_{b}(r)$. The numerical solution of this system of equations can be obtained with a generalization of the elegant algorithm for a monodisperse colloidal suspension proposed in Ref. [5].

The generalization to a mixture of $M>2$ colloidal species with radii $r_{i}$, packing fractions $\eta_{i}$ and charges $Z_{i}$, is performed by introducing $M$ cells of radii $R_{i}(i=$ $1,2, \ldots, M)$, in which the Poisson-Boltzmann equation (1) with boundary conditions (2) and (3) is to be solved. To fix the values of the $M$ radii of the cells, the equivalent of Eq. (4) is

$$
\sum_{i=1}^{M} \eta_{i} \frac{R_{i}^{3}}{r_{i}^{3}}=1
$$

Additionally, we have $M-1$ independent equations of continuity for the electric potential at the cell boundaries of the form

$$
\phi_{i}\left(R_{i}\right)=\phi_{1}\left(R_{1}\right)
$$

for $i=2, \ldots, M$. This completes the system of equations for the electric potentials and the radii of the cells. 


\section{A. Renormalized charge}

The renormalized charge, defined using Alexander prescription [3], is obtained by comparing the nonlinear solution of the Poisson-Boltzmann equation with its linearized solution, where the linearization is performed with respect to the value of the potential at the boundary of the cell. Following Ref. [5], and restricting the analysis to a binary mixture for the sake of simplicity, let us define $\tilde{\phi}_{i}(r)=\phi_{i}(r)-\phi_{0}$, for each cell $i=a, b$, with $\phi_{0}=\phi_{a}\left(R_{a}\right)=\phi_{b}\left(R_{b}\right)$. Supposing that $\tilde{\phi}_{i}$ is small, it satisfies the linearized Poisson-Boltzmann equation

$$
\Delta \tilde{\phi}_{i}(r)=\kappa_{\mathrm{PB}}^{2}\left(\tilde{\phi}_{i}(r)+\gamma_{0}\right)
$$

with $\kappa_{\mathrm{PB}}^{2}=\kappa^{2} \cosh \phi_{0}$ and $\gamma_{0}=\tanh \phi_{0}$, and the boundary conditions $\tilde{\phi}_{i}\left(R_{i}\right)=0$ and $\tilde{\phi}_{i}^{\prime}\left(R_{i}\right)=0$. The solution of the linearized problem is

$$
\begin{aligned}
\tilde{\phi}_{i}(r)= & \gamma_{0}\left[-1+\frac{e^{\kappa_{\mathrm{PB}}\left(r-R_{i}\right)}\left(\kappa_{\mathrm{PB}} R_{i}+1\right)}{2 \kappa_{\mathrm{PB}} r}\right. \\
& \left.+\frac{e^{\kappa_{\mathrm{PB}}\left(R_{i}-r\right)}\left(\kappa_{\mathrm{PB}} R_{i}-1\right)}{2 \kappa_{\mathrm{PB}} r}\right] .
\end{aligned}
$$

The linear potential $\tilde{\phi}_{i}(r)+\phi_{0}$, the behavior of which approximates the nonlinear solution $\phi_{i}(r)$ near the cell boundary, is created by an effective or renormalized charge $Z_{i}^{\text {ren }}=r_{i}^{2} \tilde{\phi}_{i}^{\prime}\left(r_{i}\right) / \lambda_{B}$ where $r_{i}$ is the colloid radius ( $r_{a}=a, r_{b}=b$ for $i=a, b$ respectively). Explicitly,

$$
\begin{aligned}
Z_{i}^{\mathrm{ren}} \frac{\lambda_{B}}{r_{i}}= & \frac{\gamma_{0}}{\kappa_{\mathrm{PB}} r_{i}}\left[\left(\kappa_{\mathrm{PB}}^{2} R_{i} r_{i}-1\right) \sinh \left(\kappa_{\mathrm{PB}}\left(R_{i}-r_{i}\right)\right)\right. \\
& \left.+\kappa_{\mathrm{PB}}\left(R_{i}-r_{i}\right) \cosh \left(\kappa_{\mathrm{PB}}\left(R_{i}-r_{i}\right)\right)\right]
\end{aligned}
$$

As mentioned before, a convenient numerical algorithm to compute the renormalized charges can be formulated on the basis of the method introduced in Ref. [5]: we impose a value $\phi_{0}$ for common value of the electric potential at the cell boundaries. Solving numerically the system of equations (1)-(5) allows us to determine the radius of the cells $R_{a}$ and $R_{b}$ and the bare charges $Z_{a}$ and $Z_{b}$ corresponding to the value $\phi_{0}$ of the potential at the boundaries of the cells. Finally, using Eq. (10) we compute the corresponding renormalized charges $Z_{a}^{\text {ren }}$ and $Z_{b}^{\text {ren }}$. The osmotic properties of the suspension can also be determined by this procedure as discussed below.

\section{B. Thermodynamic properties}

In the mean field approximation, the grand potential per cell $\left(\omega_{a}\right.$ or $\left.\omega_{b}\right)$ is given by (see e.g. Ref [18])

$$
\begin{aligned}
\beta \omega_{i}= & \frac{1}{8 \pi \lambda_{B}} \int\left|\nabla \phi_{i}(r)\right|^{2} d \mathbf{r} \\
& +\int \sum_{\alpha= \pm}\left[\rho_{i}^{\alpha}(r)\left(\ln \frac{e^{\beta \mu_{0}} \rho_{i}^{\alpha}(r)}{\rho_{s}}-1\right)\right] d \mathbf{r} \\
& -\int \beta \mu_{0}\left[\rho_{i}^{+}(r)+\rho_{i}^{-}(r)-2 \rho_{s}\right] d \mathbf{r},
\end{aligned}
$$

where the ionic density profiles inside each cell are given by

$$
\rho_{i}^{ \pm}(r)=\rho_{s} e^{\mp \phi_{i}(r)} .
$$

The integration is performed in the cells excluding the volume occupied by the colloid, i.e., $r_{i}<r<R_{i}$.

Using Gauss' law, Eqs (2) and (3), this can be written as

$$
\begin{aligned}
\beta \omega_{i} & =\frac{Z_{i}}{2} \phi_{i}\left(r_{i}\right) \\
& +4 \pi \rho_{s} \int_{r_{i}}^{R_{i}} d r r^{2}\left[\phi_{i}(r) \sinh \phi_{i}(r)-2 \cosh \phi_{i}(r)\right] .
\end{aligned}
$$

The total grand potential of the system is

$$
\Omega=N_{a} \omega_{a}+N_{b} \omega_{b}+F_{a}^{i d}+F_{b}^{i d}
$$

where $\beta F_{i}^{i d}=N_{i}\left[\ln \left(\rho_{i} \Lambda_{i}^{3}\right)-1\right]$ accounts for the colloid entropy, with $\Lambda_{i}$ and $N_{i}$ the thermal length of colloids of species $i$ and their number respectively. The electrostatic contribution to the pressure $p$ can be obtained deriving $\Omega$ with respect to the volume $V$. Introducing the osmotic pressure $\Pi=p-p_{\text {res }}$ with $p_{\text {res }}$ the pressure in the electrolyte reservoir, the final result can be expressed in terms of the potential at the boundary of the cells $\phi_{0}$ in the form

$$
\frac{\beta \Pi}{\rho}=1+\frac{2 \rho_{s}}{\rho}\left(\cosh \phi_{0}-1\right)
$$

with $\rho=\rho_{a}+\rho_{b}$ the total number density of colloids. The l.h.s. of Eq (15) defines the osmotic compressibility factor which equals one in the dilute limit. In the next section we apply the model formulated above to study charge renormalization and osmotic properties in a colloidal binary mixture.

\section{COLLOIDAL BINARY MIXTURE}

Let us consider a colloidal mixture consisting of two species of colloids. In order to reduce the number of parameters, we will assume that the colloids have the same radius, i.e., $a=b$ and we will express all lengths in units of this quantity. In addition, the Bjerrum length will be fixed to $\lambda_{B} / a=0.0022$ which is a typical value for a suspension of colloidal particles in water. The remaining free parameters are (i) the bare charge of species $a: Z_{a}$, (ii) the bare charge of species $b: Z_{b}$ (iii) the total packing fraction: $\eta$, (iv) the composition of the mixture: $x$, and $(\mathrm{v})$ the concentration of electrolyte as given by the parameter $\kappa a$, where $\kappa^{-1}$ is the screening length of the electrolyte reservoir. Notice that the screening parameter $\kappa$ is different from $\kappa_{P B}$ as defined after equation (8), the later corresponding to the electrolyte concentration at the cell boundary. 


\section{A. Renormalized charges}

Let us start our analysis by considering the behavior of the renormalized charges of the binary mixture as a function of the structural charges. Fig. 1 shows the renormalized charge for each species when the bare charge of one of the species is fixed while that of the other species is changed. In Fig. 1a the structural charge of species $b$ is fixed to $Z_{b} \lambda_{B} / a=5$ while $Z_{a}$ varies. In Fig. $1 \mathrm{~b}$ we fixed $Z_{a} \lambda_{B} / b=10$ and vary the bare charge of species $b$. Two different compositions are considered, $x=0.05$ and $x=0.95$, and two screening lengths $\kappa a=0.1$ and $\kappa a=1$. The remaining parameters are fixed to $\eta=0.1$, $\lambda_{B}=0.72 \mathrm{~nm}$ and $a=b=326 \mathrm{~nm}$. In Fig. 2 a similar study is performed, this time for a larger total packing fraction of the mixture $\eta=0.3$. A first observation following Figs. 1 and 2 is that the dependence of $Z_{i}^{\text {ren }}$ is similar to the behavior found in monodisperse systems: as the bare charges $Z_{i}$ increases, the corresponding renormalized charges $Z_{i}^{r e n}$ grows. The growth is only linear for small values of the varying structural charges. For larger values of $Z_{i} \lambda_{B} / a$ the increment becomes highly nonlinear (compared to the dashed lines corresponding to $Z_{i}^{r e n}=Z_{i}$ ) and saturates towards a value much smaller than the structural charge for $Z_{i} \lambda_{B} / a \gg 1$. Notice that the saturation value of the charges depends not only on the total packing fraction but also on the composition of the mixture. The influence of the electrolyte concentration on the saturation curves, on the other hand, is relatively small, as compared to the effect produced by a drastic change in composition or total packing fraction of the mixture. In particular, in Fig. 2 the curves corresponding to $\kappa a=1$ and $\kappa a=0.1$ are indistinguishable. For the packing fractions considered in these examples, reducing the electrolyte concentration manifest itself in a slight reduction of the saturation values in all cases.

The concentration of colloidal particles in suspensions can be adjusted and measured in a wide range of packing fractions, therefore, it is interesting to analyze to what extent the general behavior of the renormalized charges described above depends on this quantity. In Fig. 3 we consider the scaled renormalized charges corresponding to a total packing fraction varying over several decades. The bare charges of species $a$ and $b$ are fixed by $Z_{a} \lambda_{B} / a=10$ and $Z_{b} \lambda_{B} / a=5$ respectively. The figure shows curves obtained for different electrolyte concentrations as fixed by the parameter $\kappa a$. Several values of $x=\eta_{a} / \eta$ are represented by different types of lines. For $\kappa a \gtrsim 1$ and $\eta \lesssim 0.01$ varying either the electrolyte concentration, the total packing fraction, or the composition of the mixture has a small effect on the values of the renormalized charges. For $\eta \gtrsim 0.01$ varying the composition of the mixture causes the charge renormalization curves to split into several branches. This effect is also present for smaller total packing fractions in the extremely low electrolyte concentration curve corresponding to $\kappa a=0.1$. In the latter case, the values of the renormalized charges are much more sensitive to
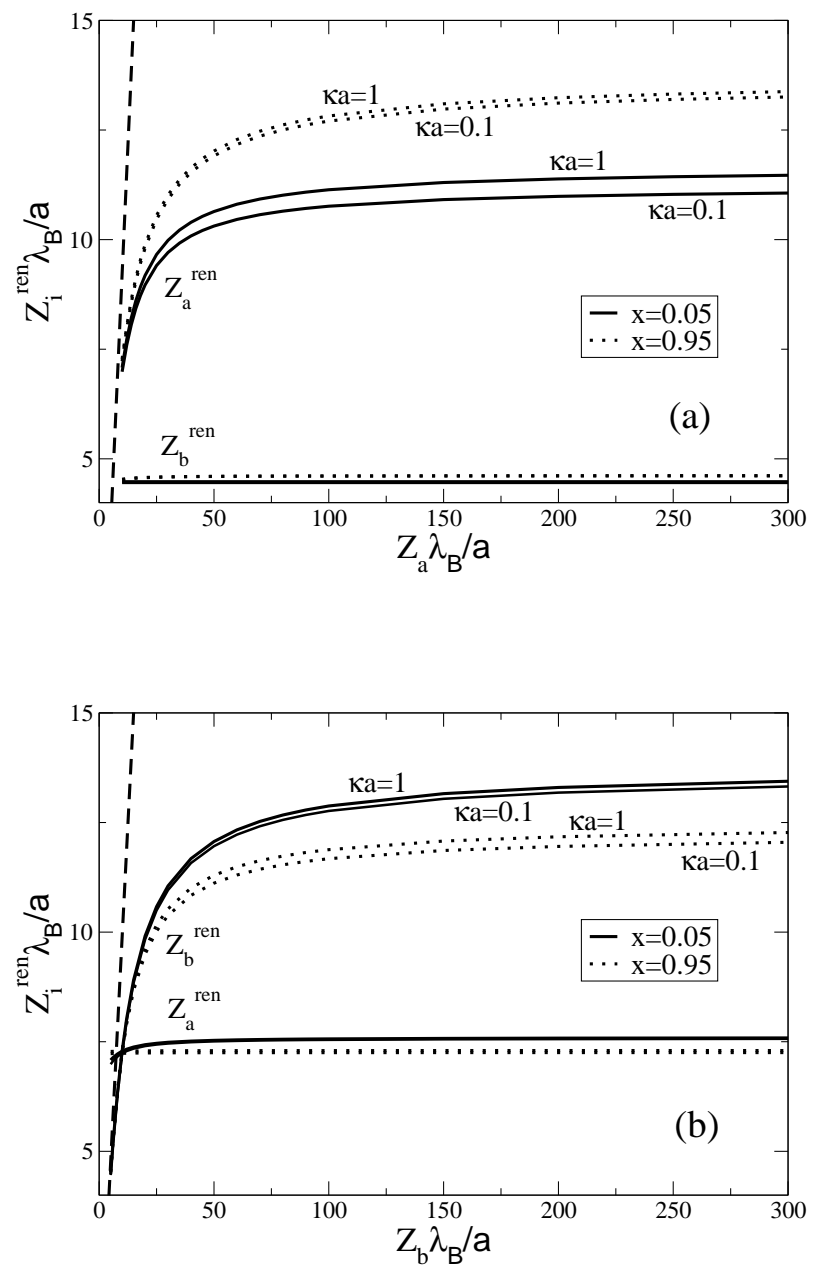

FIG. 1: Renormalized charges $Z_{a}^{r e n} \lambda_{B} / a$ and $Z_{b}^{r e n} \lambda_{B} / a$ for a binary mixture with: (a) fixed bare charge $Z_{b} \lambda_{B} / a=5$ and varying bare charge $Z_{a} \lambda_{B} / a$ and (b) fixed bare charge $Z_{a} \lambda_{B} / a=10$ and varying bare charge $Z_{b} \lambda_{B} / a$ for $\lambda_{B}=$ $0.72 \mathrm{~nm}$ and $a=b=326 \mathrm{~nm}$. The total packing fraction is fixed to the value $\eta=0.1$. Different electrolyte concentrations and compositions are considered as pointed by the legends. For the sake of comparizon, the dashed line shows the linear dependence corresponding to the case $Z_{i}^{r e n}=Z_{i}$.

variations of the total packing fraction, which increases after reaching a minimum at about $\eta=0.015$. At total packing fractions $\eta \gtrsim 0.2$ all the curves show the same qualitative behavior and tend to merge into lines of similar slope.

In Fig. 4 we show the effect of varying the composition of the mixture on the scaled renormalized charge of species $a$. We observe that $Z_{a}^{r e n} \lambda_{B} / a$ varies linearly with changing $x$ between the corresponding values of a pure species $-a$ system, i.e., $x=1$ and a pure species $-b$ system, i.e., $x=0$. Similar curves are obtained for 

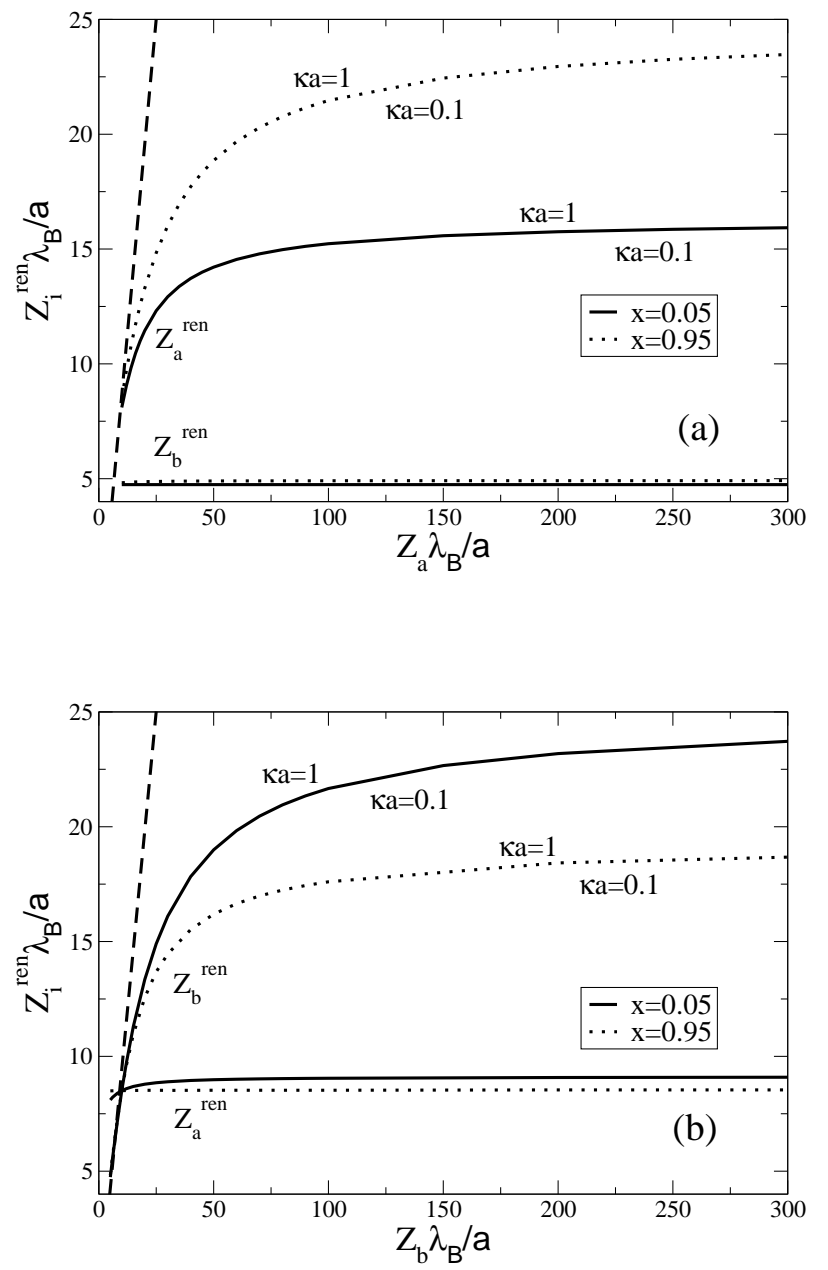

FIG. 2: Renormalized charges for a binary mixture as given in Fig. 1. Here we consider a higher value of the total packing fraction, $\eta=0.3$.

$Z_{b}^{r e n} \lambda_{B} / a$. The slope of the renormalized charge curves is slightly larger for small electrolyte concentration and is practically zero for $\kappa a=2$. For the larger packing fractions (triangles), the general tendency is that the renormalized charges increase with $x$. For smaller packing fractions and low electrolyte concentration (squares in Fig. 4a), this tendency is reversed in correspondence with the behavior observed in Fig. 3a.

\section{B. Osmotic properties}

In Fig. 5 we show the compressibility factor (Eq. (15)) as a function of the total packing fraction, corresponding to the four different electrolyte concentrations considered in Fig 3. Different types of lines illustrate the
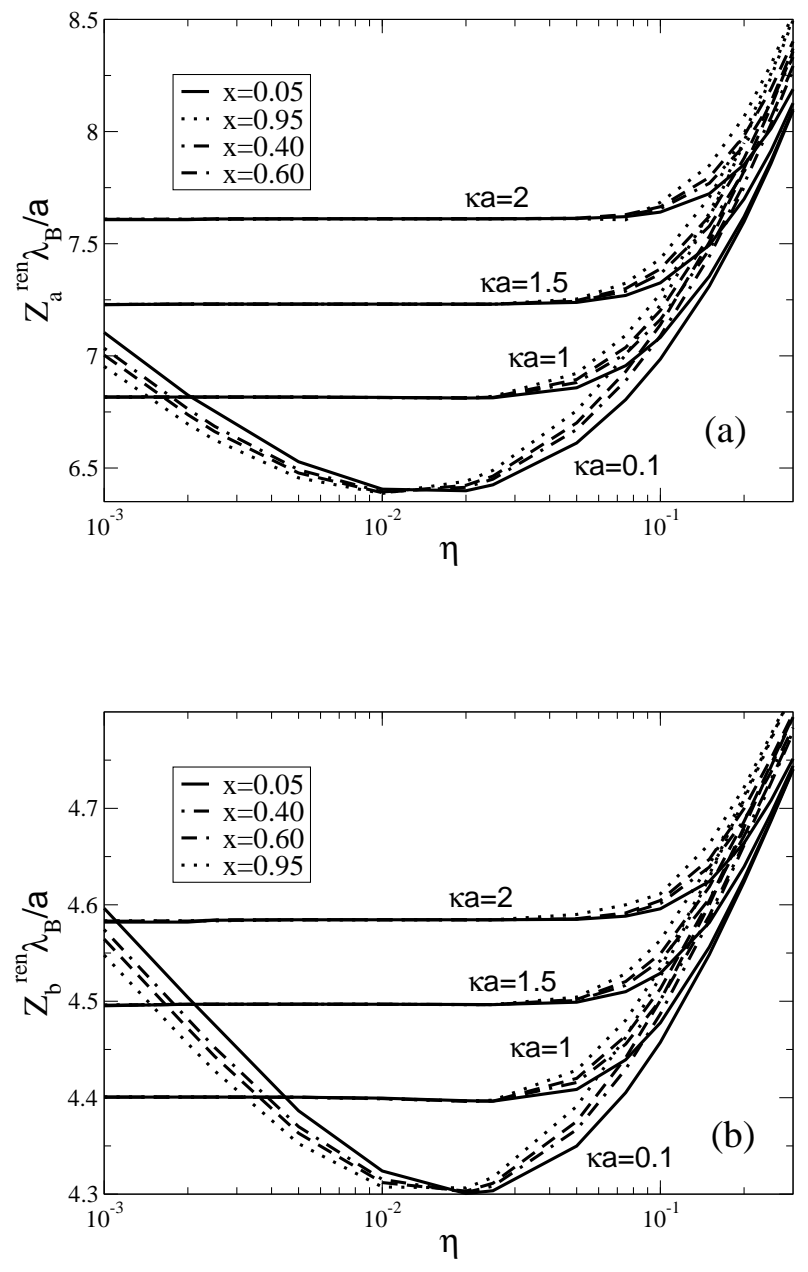

FIG. 3: Renormalized charges $Z_{a}^{r e n} \lambda_{B} / a$ and $Z_{b}^{r e n} \lambda_{B} / a$ for a binary mixture with bare charges $Z_{a} \lambda_{B} / a=10$ and $Z_{b} \lambda_{B} / a=5$ as a function of the total packing fraction $\eta$. Different electrolyte concentrations as fixed by the parameter $\kappa a$ are shown. The values of the remaining parameters are typical for colloidal suspensions, namely $\lambda_{B}=0.72 \mathrm{~nm}$ and $a=b=326 \mathrm{~nm}$. Different types of lines correspond to distinct compositions of the mixture as indicated by the legends.

effect of varying the compositions of the mixture. In general, adding particles of species with the larger charge increases the compressibility factor. This effect is particularly notorious at high packing fractions. At low packing fractions the electrostatic pressure reduces to the value of the reservoir pressure and the compressibility factor decays towards the ideal gas value, i.e., $\beta \Pi / \rho=1$. It is interesting to observe the strong effect of varying the electrolyte concentration: following the sequence from Fig. 5a to Fig. 5d one notices that the compressibility factor decreases by about two orders of magnitude for $\eta<0.1$. This diminishing in the compressibility factor is 


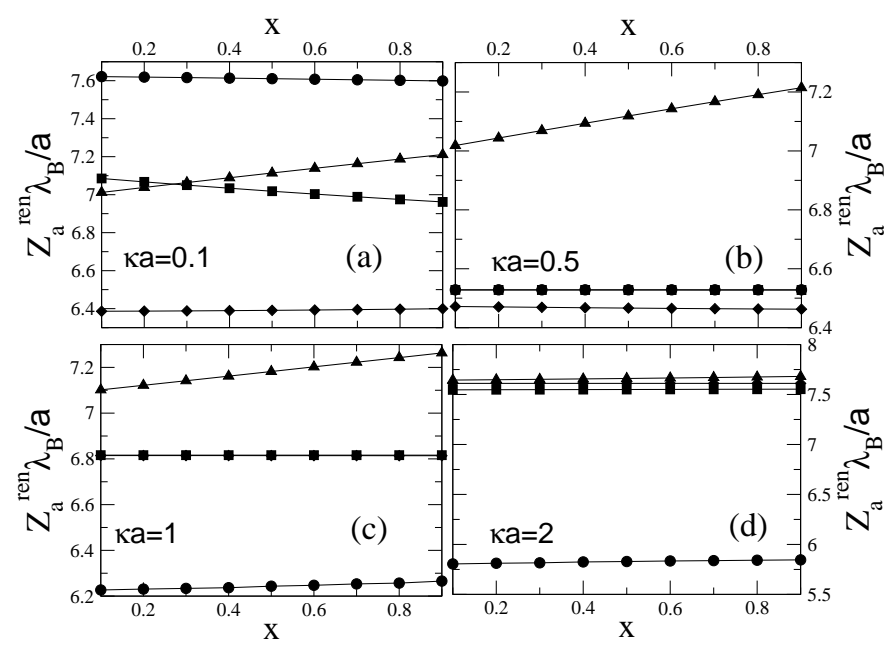

FIG. 4: Renormalized charge $Z_{a}^{r e n} \lambda_{B} / a$ as a function of the composition $x=\eta_{a} / \eta$ for different salt concentrations for the parameters $b=a, Z_{a} \lambda_{B} / a=10$, and $Z_{b} \lambda_{B} / a=5$. We consider different values of the total packing fraction, namely $\eta=10^{-4}$, (circles), $\eta=10^{-3}$, (squares), $\eta=10^{-2}$ (diamonds), and $\eta=10^{-1}$ (triangles).

a consequence of the decreased range of the repulsions. Moreover, for low electrolyte concentrations the compressibility factor rapidly grows as a function of the total packing fraction. This effect is intensified by increasing the concentration of the highest charged species, species $a$ in this particular case study. For higher electrolyte concentrations, Fig. 5d, the rate of change of the compressibility factor is reduced and it assumes smaller values as comparison with Fig. 5a shows, i.e., the system becomes ideal-like in the dilute regime, as a consequence of the screening of the strong repulsive electrostatic forces. This can be seen more clearly from the osmotic equation of state shown in Fig. 6 for several electrolyte concentrations. In Fig. 6a, corresponding to extremely low screening, the osmotic pressure assumes large values and the suspension is expected to be in crystal phase. After increasing the screening constant, the osmotic pressure assumes values close to zero which means that the excess pressure due to electrostatic interactions reduces to the reference pressure in the reservoir.

\section{Cell radii}

In Fig. 7 we plot the cell radii (expressed in units of the colloidal radius $a$ ) as a function of the charge of one of the species while the charge of the other species is fixed (see details in the caption). In general, as one of the radii increases the other radius decreases to satisfy the constraint of fixed total packing fraction as expected from Eq. (4). The form in which this occurs depends

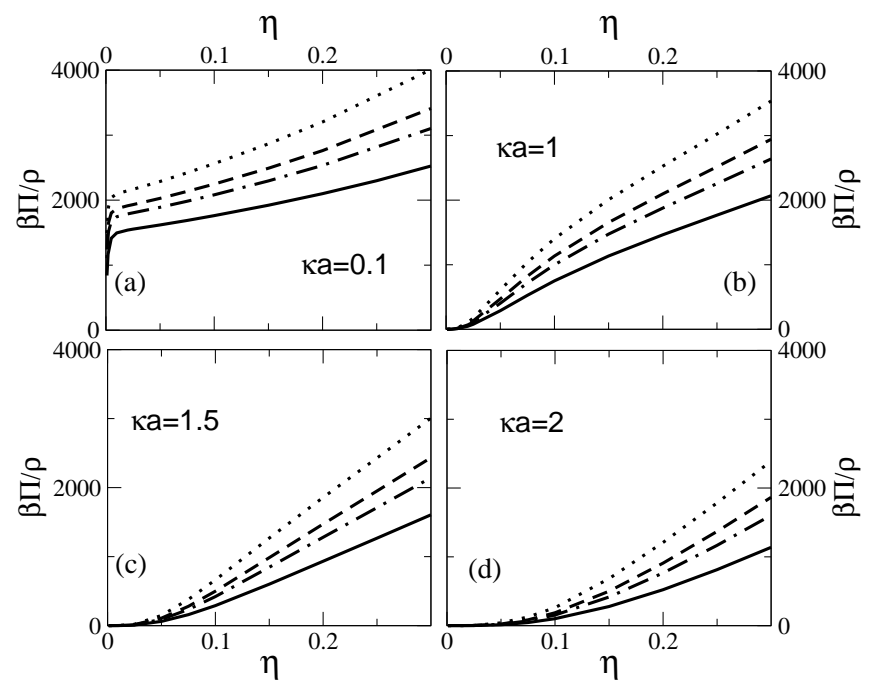

FIG. 5: Compressibility factor as obtained from Eq. (15) as a function of the total packing fraction for different electrolyte concentrations. The line-type convention for different compositions the same as in Fig. 3.

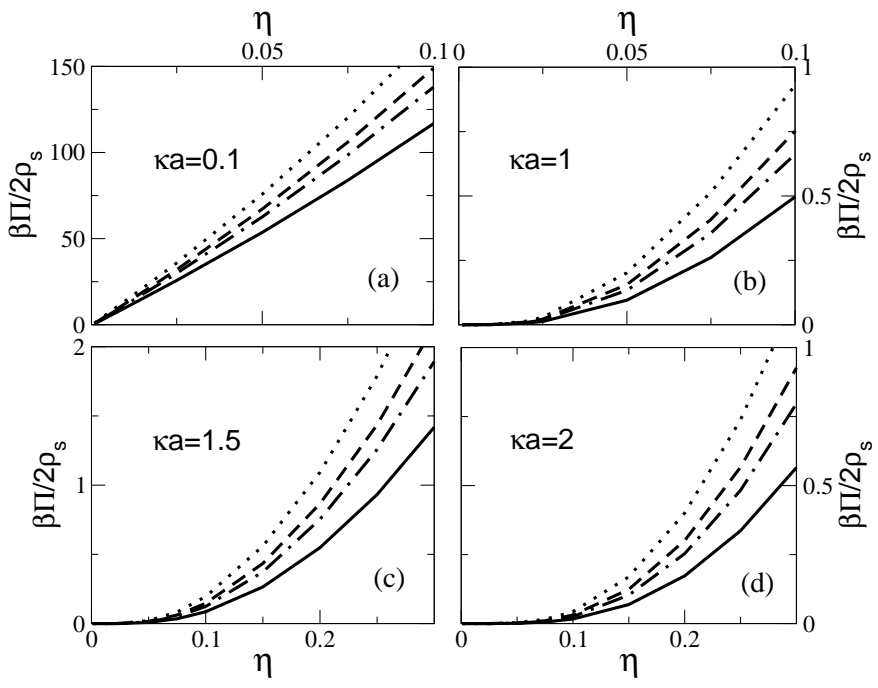

FIG. 6: Osmotic pressure as a function of the total packing fraction for different electrolyte concentrations. The line-type convention is the same as in Fig. 3.

on the colloid charges and compositions as well as on the other physicochemical parameters in the model. A small total packing fraction allows for larger cell radii as we notice by comparing Figs. 7a and 7c on the one hand and Figs. $7 \mathrm{~b}$ and $7 \mathrm{~d}$ on the other hand. Notice also that in the latter case for small values of $Z_{b} \lambda_{B} / a$ we have $R_{a} / R_{b}>1$. After the charge of species $b$ is increased, the inequality quickly inverts i.e., the volume of the cells surrounding the now highly charged particles of species $b$ 

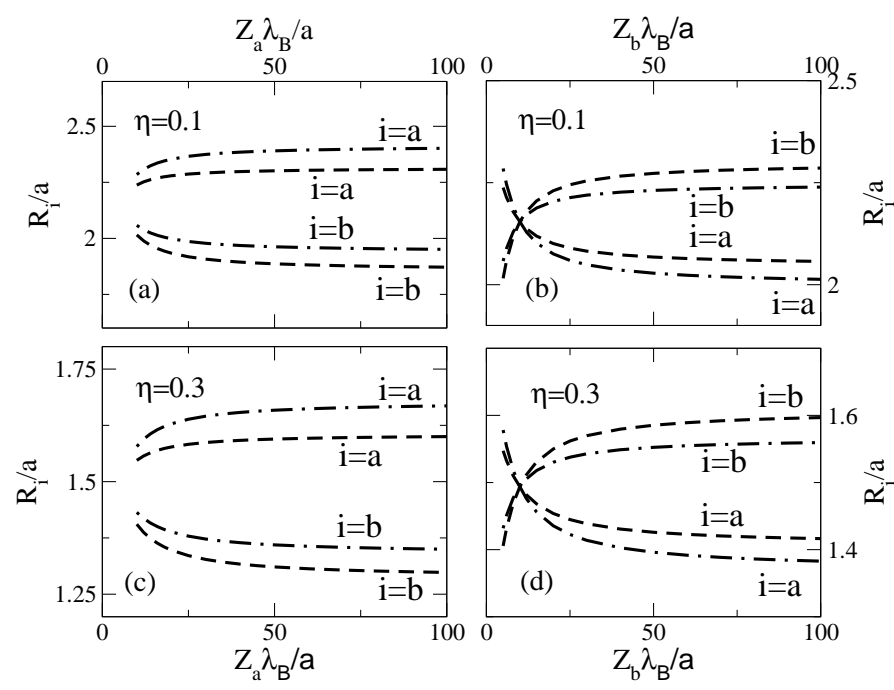

FIG. 7: Radii of the cells $R_{a}$ and $R_{b}$ surrounding particles of type $a$ and $b$ respectively expressed in units of the colloids radius $a=b=326 \mathrm{~nm}$. In (a) and (c) the bare charge of type-a particles varies while $Z_{b} \lambda_{B} / a=5$ is kept fixed. In (b) and (c) the bare charge of type-b particles varies while $Z_{a} \lambda_{B} / a=10$ remains unchanged. Two different total packing fractions are considered as pointed by the legends and the values $\lambda_{B}=0.72 \mathrm{~nm}$ and $\kappa a=1$ are used in all figures. The line-type convention is the same as that in Fig. 3.

surpasses that of species $a$. Finally, it is worth to notice the fact that beyond some value of $Z_{i} \lambda_{B} / a$ the radii of the cells become almost charge independent, reflecting the fact that the renormalized charges have reached their saturation value. In the light of the previous case study, we now proceed to discuss in more detail some general features of the polydisperse cell model.

\section{DISCUSSION}

Physically, the charge renormalization process results from the accumulation of ions in the vicinity of a highly charged object immersed in an electrolyte. Therefore, the relevant parameter to compute their interactions is not the bare charge but the effective (renormalized) charge that takes into account this non-linear screening effect. As shown in Fig. 1, the behavior of the renormalized charges for polydisperse systems at the level of PB theory is reminiscent of that of their monodisperse counterpart: the renormalized charges saturate to a finite value when the structural charge becomes sufficiently large. The saturation values can be orders of magnitude smaller than the structural charges. As a consequence of this non-linear screening effect, a very asymmetric colloidal mixture behaves as a much more symmetric system as regards their electrostatic effects. For instance, from Fig. $2 \mathrm{~b}$ one can notice that a colloidal binary mix- ture with charges $Z_{a} \lambda_{B} / a=10$ and $Z_{b} \lambda_{B} / a=300$ behaves effectively as a mixture with $Z_{a}^{r e n} \lambda_{B} / a \simeq 7.5$ and $Z_{b}^{r e n} \lambda_{B} / a \simeq 13$, so that the ratio $Z_{a} / Z_{b}=0.03$ changes to $Z_{a}^{r e n} / Z_{b}^{r e n}=0.58$ which corresponds to a much more charge symmetric effective mixture. On the other hand, for small values of the structural charges, $Z_{i}^{r e n} \simeq Z_{i}$. As a consequence, expressions for the effective interaction potentials derived from linear theories, e.g., DLVO theory, are expected to be sufficient to describe the thermodynamic properties of the suspension, at least in the regime where pairwise additivity is expected $[19,20]$.

The saturation values of the renormalized charges depend on the physicochemical parameters: the electrolyte concentration, total colloids density, the dielectric constant of the solvent and the composition of the mixture. Increasing the electrolyte concentration has the effect of enhancing the value of the renormalized charges. This effect induces two competing tendencies in the screened interaction potential. On one hand the spatial extent of the double-layer reduces, on the other hand the amplitude of the interactions increases. This tendency is inverted in the case of weak screening and total colloids packing fraction smaller that about $5 \%$ (see Fig. 3 ). The balance of these two non-linear screening features is affected by the composition of the mixture and is expected to have consequences on, e.g., the phase diagrams of mixtures of highly charged macroions computed by using Yukawa-like potentials, the omission of such non-linear screening effects, as accounted by $Z_{i}^{r e n}$, may lead to unphysical results as demonstrated by several authors (See e.g. $[21,22])$.

In the context of the cell model for monodisperse systems, it is assumed that due to entropic reasons, the colloidal particles tend to occupy the maximum space possible inside the solvent. Therefore, it is feasible to factorize the suspension into spherical cells, each of them housing a colloidal particle in its center. The radius of such cells is then chosen in such a fashion that the cells fill all the suspension, namely $R / a=\eta^{-1 / 3}$. In other words, the packing fraction alone determines the size of the cells. In contrast, in the polydispense cell model, the radii of the cells depend not only on the total packing fraction and the composition of the mixture according to Eq. (4), but also on the relative charges of the species through the boundary conditions expressed by Eqs. (3) and (5). In other words, the volumes of the cells are not only determined by fixing the total packing fraction, but adapt themselves in order to satisfy the boundary conditions which impose the cells to be electrically neutral, the potential to be continuous at the cells boundaries and the cells to fill the whole volume accessible to the colloidal particles. The resulting cells are independent units regarding electrostatic interactions in virtue of their neutrality. Therefore, the grand potential of the entire suspension can be easily calculated as the sum of the grand potential of individual cells. This facilitates enormously the calculation of thermodynamic response functions, in particular, following the contact theorem, 
(see e.g., Ref. [2]), the osmotic pressure can be obtained directly from the value of the electrostatic potential at the cells boundary as explained in Sec. 2. The value of the potential at the cell boundary depends on the size of the cell, which have a priori different radii depending on the parameters, and in particular, on the composition of the mixture.

Experimentally, in spite of the recent advances in electrophoretic techniques [23], it is difficult to precisely measure the values of renormalized charges in colloidal suspensions. Therefore, it would presumably be intricate to collect experimental evidence of, e.g., the splitting of the charge renormalization curves in Fig. 3, even at the highest packing fractions. Osmotic properties are nevertheless relatively easy to probe experimentally e.g., in sedimentation-diffusion equilibrium [24],[25]. In that sense the present formalism provides a reasonably simple and numerically robust scheme for the calculation of the osmotic equation of state and compressibility factors in a wide range of parameters. As illustrated in the case study of Sec. 2, these osmotic properties are non-trivially dependent on the mixture composition, which illustrates the fact that the relatively small change in the values of the corresponding renormalized charges shown in Fig. 3, do not necessarily imply a mild change in the response functions: the former are obtained from changes in the slope of the potential at the colloids surface as given by the solution of the linearized PB equation (see Eq. 10), whereas the latter depend on the value of the potential at the cells boundary (Eq. 15). The screening parameter, however, plays a much more important role in the osmotic properties than the relative composition of the mixture, and may cause, for instance, the osmotic pressure to vary over several orders of magnitude.

It is worth to mention the fact that in the polydisperse cell model we restricted the attention to colloids of the same sign of charge. The reason is that one can impose on a physical ground that each cell surrounding a colloid is charge neutral i.e., $\phi_{i}^{\prime}(R)=0$ for $i=1, \ldots, M$. It is not obvious that such a condition can be imposed for oppositely charged colloids, as the potential may change sign in between the colloids while the electric field does not vanish anywhere. The attractive or repulsive nature of the interactions between the cells is not obvious to estimate by analyzing the sign of the potential, since e.g., potentials opposite in sign can still lead to colloidal repulsions [26]. A second limitation related to the cells electoneutrality, which is also present in Alexander's formulation, is that as a consequence of the restriction of the theoretical analysis to a single cell per colloidal component, all information on correlations between the colloids is lost. This may lead to unphysical ideal-gas-like values for the osmotic pressure in highly screened suspensions [27]. Care has to be exercised in drawing conclusion from cell models in this regime.

An important feature of charge renormalization is the fact that increasing the electrolyte concentration leads to higher values of the renormalized charges simultaneously reducing the extension of the double-layer [6, 7]. These two non-linear screening features are neglected in linear theories, such as the DLVO model, where the (structural) charge is in principle independent of the screening parameter. These artifacts of the linearization procedure can be cured by using the renormalized values of the charges within the linear schemes, which can in principle be achieved for example by using the analytical solution of the Ornstein-Zernike equation for a mixture interacting via a DLVO-like potential within Mean Spherical Approximation, by replacing $Z_{i} \rightarrow Z_{i}^{r e n}$. A second alternative is provided by the variational treatment of DLVOlike potentials based on the Gibbs-Bogoliubov inequality, which uses the Laplace transforms of the pair correlation functions to estimate the free energy of the system though a numerical optimization problem (see e.g., Refs. $[28,29])$. This alternative is extremely suitable for the calculation of phase diagrams by e.g., using Maxwell constructions, providing a simple alternative for the study of phase behavior of highly charged colloidal mixtures while fully taking into account non-linear effects.

\section{CONCLUSION}

In this paper we proposed a simple model which allows to study the thermodynamic properties of mixtures of highly charged macroions suspended in an electrolyte. The model is a generalization of the well-know cell model for colloids, and the notion of charge renormalization as introduced by Alexander and collaborators [3]. In contrast to the one-component cell model, where the radius of the cells is fixed by the packing fraction of colloids, in the polydisperse cell model the radii of the cells depend on the physicochemical parameters through the boundary conditions utilized to solve the non-linear PoissonBoltzmann equation in the cell geometry. The radii of the cells play the role of Lagrange multipliers that enforce the constraint that the cells fill the entire volume occupied by the colloidal mixture and have to be determined within the numerical solution of the non-linear differential equations. We presented a method to perform such a calculation based on the algorithm of Ref. [5], which also allows to determine thermodynamic response functions and renormalized charges within a clear and efficient numerical scheme. We presented a detailed example of such an analysis in the case of a colloidal binary mixture, where we studied the effect that several physicochemical parameters have on the values of the saturation curves for the renormalized charges and osmotic properties of the suspension. In general, the ion condensation phenomenon that leads to charge renormalization has the effect of making the mixture more "electrostatically symmetric", in the sense that the ratio of the renormalized charges is much closer to one than that of the structural charges. Increasing the concentration of the species with the greatest charge keeping the total packing fraction fixed, raises the osmotic pressure. An important limi- 
tation of the present model, which is actually shared by all Poisson-Boltzmann-based models, is the fact that ionion correlations are neglected. This oversimplification of the problem may lead not only to quantitative but also qualitative modifications to the saturation curves: the renormalized charge may exhibit a maximum followed by a decrease of $Z_{i}^{r e n}$ with $Z_{i}$ increasing (see e.g., Ref. [30-32]). In some cases this can lead to a weakening of the repulsive effective interactions as calculated on the basis of the renormalized charges, which may eventually turn electrostatic repulsions into attractions. In the case of 1:1 electrolytes, such ion-ion correlations are relatively unimportant and the present Poisson-Boltzmann model is expected to provide a reasonably good description of highly charged colloidal mixtures.

\section{Acknowledgments}

This work is part of the research program of the Stichting voor Fundamenteel Onderzoek der Materie (FOM), which is financially supported by the Nederlandse Organisatie voor Wetenschappelijk Onderzoek (NWO).
[1] Verwey, E. J. W.; Overbeek, J. Th. G. Theory of the stability of lyophobic colloids Elsevier: Amsterdam, 1948.

[2] J. N. Israelachvili, Intermolecular and Surface Forces: With Applications to Colloidal and Biological Systems. Academic Press, 1985.

[3] S. Alexander, P. M. Chaikin, P. Grant, G. J. Morales, and P. Pincus, J. Chem. Phys. 80, 5776 (1984).

[4] R. Kjellander and D.J. Mitchell J. Chem. Phys. 101, 603 (1994).

[5] E. Trizac, L. Bocquet, M. Aubouy, H. H. von Grunberg, Langmuir 19, 4027 (2003).

[6] E. Trizac, L. Bocquet, and M. Aubouy, Phys. Rev. Lett. 89, 248301 (2002).

[7] M. Aubouy, E. Trizac, and L. Bocquet, J. Phys. A: Math. Gen. 36, 5835 (2003).

[8] M. Quesada-Pérez, J. Callejas-Fernández and R. Hidalgo-Álvarez, J. Coll. Interface Sci. 206, 354 (1998).

[9] P. Wette, H.J. Schöpe and T. J. Chem. Phys. 116, 10981 (2002).

[10] A.-P. Hynninen and M. Dijkstra, Phys. Rev. Lett. 94, 138303 (2005).

[11] A.-P. Hynninen, C.G. Christova, R. van Roij, A. van Blaaderen, and M. Dijkstra, Phys. Rev. Lett. 96, 138308 (2006).

[12] J. Baumgartl, R.P.A. Dullens, M. Dijkstra, R. Roth, and C. Bechinger, Phys. Rev. Lett. 98, 198303 (2007).

[13] M. Leunissen et. al., Nature. 437, 235 (2005).

[14] J. Dzubiella et. al., Phys, Rev. E. 65, 021402 (2002).

[15] M. Rex and H. Löwen, Phys, Rev. E. 75, 051402 (2007).

[16] J. Zwanikken and R. van Roij, Europhys. Lett. 71, 480 (2005).
[17] A. Esztermann and H. Löwen Europhys. Lett. 68, 120 (2004); M. Dijkstra, J. Zwanniken, and R. van Roij, J. Phys.: Condens. Matter, 18, 825 (2006).

[18] H. H. von Grünberg, R. van Roij, and G. Klein. Europhys. Lett. 55, 580 (2001).

[19] L. Belloni, J. Chem. Phys. 123204705 (2005).

[20] A. Torres, A. Cuetos, M. Dijkstra, R. van Roij, et al., Phys. Rev. E 75, 041405 (2007).

[21] Y. Levin, M. C. Barbosa and M. N.Tamashiro, Europhys. Lett. 41, 123 (1998).

[22] M. N. Tamashiro, Y. Levin and M. C. Barbosa, Physica A 258, 341 (1998)

[23] V. Lobaskin et al., Phys. Rev. Lett. 98, 176105, (2007).

[24] R. Piazza, T. Bellini and V. Degiorgio, Phys. Rev. Lett. 71, 4267 (1993).

[25] C.P. Royall, R. van Roij and A. van Blaaderen, J. Phys.: Cond. Matt. 17, 2315 (2005).

[26] A. Torres and R. van Roij, Langmuir (2008) in press.

[27] M. Deserno and H. H. von Grünberg, Phys. Rev. E 66, 011401 (2002).

[28] T. Boublík, I. Nezbeda and K. Hlavatý, Statistical thermodynamics of simple liquids and their mixtures. Elsevier, 1980.

[29] Y. Rosenfeld, Phys. Rev. E, 54, 2827 (1996)

[30] L. Belloni Colloids Surfaces A, 140, 227 (1998).

[31] D. Leger and D. Levesque, J. Chem. Phys. 123, 124910 (2005).

[32] M. Camargo and G. Téllez, arxiv.org:cond-mat/0702056 (2007). 